\title{
Nucleoplasty in the Treatment of Lumbar Diskogenic Back Pain: One Year Follow-Up
}

\author{
Salvatore Masala, ${ }^{1}$ Francesco Massari, ${ }^{1}$ Sebastiano Fabiano, ${ }^{1}$ Antonio Ursone, ${ }^{1}$ \\ Roberto Fiori, ${ }^{1}$ Francesco Pastore, ${ }^{2}$ Giovanni Simonetti ${ }^{1}$ \\ ${ }^{1}$ Department of Diagnostic Imaging and Interventional Radiology, University of Rome "Tor Vergata”, Rome, Italy \\ ${ }^{2}$ Department of Neurosurgery, University of Rome "Tor Vergata", Rome, Italy
}

\section{Abstract}

Purpose: The spine is an important source of pain and disability, affecting two thirds of adults at some time in their lives. Treatment in these patients is mainly conservative medical management, based on medication, physical therapy, behavioral management, and psychotherapy, surgery being limited to elective cases with neurologic deficits. This study was carried out to evaluate the efficacy of percutaneous nucleoplasty in patients affected by painful diskal protrusions and contained herniations.

Methods: From February 2004 to October 2005, 72 patients (48 men, 24 women; mean age 48 years) affected by lumbar disk herniation were treated with nucleoplasty coblation. All patients were evaluated clinically and with radiography and MRI in order to confirm the presence of lumbalgic and/or sciatalgic pain, in the absence of major neurologic deficit and with lack of response after 6 weeks of conservative management.

Results: Average preprocedural pain level for all patients was 8.2 (on a visual analog scale of 1 to 10), while the average pain level at 12 months follow-up was 4.1. At the 1 year evaluation, $79 \%$ of patients demonstrated a statistically significant improvement in numeric pain scores $(p<0.01)$ : $17 \%$ (12 patients) were completely satisfied with complete resolution of symptoms, and $62 \%$ (43 patients) obtained a good result.

Conclusion: Our data indicate that nucleoplasty coblation is a promising treatment option for patients with symptomatic disk protrusion and herniation who present with lumbalgic and/or sciatalgic pain, have failed conservative therapies, and are not considered candidates for open surgery.

Correspondence to: S. Masala, MD, Department of Diagnostic Imaging and Interventional Radiology, "Tor Vergata" University General Hospital, 81 Oxford Street, 00133 Rome, Italy; email: salva.masala@tiscali.it
Key words: Diskogenic back pain-Interventional radiology—Lumbar pain—Nucleoplasty—Spine

The spine is an important source of pain, suffering, and disability, affecting two thirds of adults at some time in their lives [1, 2]. In these individuals the treatment remains mainly conservative, including medication, physical therapy, behavioral management, and psychotherapy.

In recent years the general trend in spinal surgery has been toward reduction and minimalization. In fact minimally invasive techniques for the treatment of degenerative pathology of the spine have come to be preferred by surgeons since the destructive effect on bony structures is eliminated and scar formation is greatly reduced [3]. Nucleoplasty is new minimally invasive therapeutic option that has been used for spinal procedures since July 2000 .

The following study was carried out to evaluate the efficacy of nucleoplasty, performed under local anesthesia, in patients affected by diskal protrusions and contained herniations.

\section{Materials and Methods}

From February 2004 to October 2005, a total of 72 patients (48 men, 24 women; age range 32-64 years, mean 48 years) affected by lumbar diskal protrusions and contained herniations were treated with percutaneous nucleoplasty. All patients were evaluated by radiography and MRI in order to confirm the presence of contained disk herniation.

Patients had to satisfy specific inclusion and exclusion criteria to be enrolled. Inclusion criteria were the presence of lumbalgic and/or sciatalgic pain due to diskal protrusions and contained herniations, absence of major neurologic deficit, and lack of response after 6 weeks of conservative management $[4,5]$. Conservative care consisted of the use of posture and activity modifications, physical therapy focusing on lumbar stabilization exercises, and oral nonsteroidal anti-inflammatory drugs. The exceptions to this rule were instances where the pain was func- 
tionally incapacitating and refractory to the use of oral narcotics [6]. Exclusion criteria were the presence of secondary gain issues, litigation, heavy opioid usage, and uncontrolled psychological disorders.

Patients presenting a sequestered or extruded disk herniation, a contained herniation that was larger than one third the sagittal diameter of the spinal canal, severe degenerative disk with greater than $33 \%$ loss of disk height, non-qualifying results on provocative diskography, marked spinal stenosis due to extensive osteophytosis, previous spinal surgery in the same region, spondylolisthesis, bone congenital abnormalities, evidence of infection, cauda equina syndrome, tumor or spinal instability are not considered candidates for this procedure [3-7].

A total of 72 patients were included in the study. Two patients were lost to follow-up, and results were obtained in 70 patients. Considering the main symptoms, the distribution of primary pain was mostly lumbalgic in 53 patients $(74 \%)$, mostly sciatalgic in 11 patients $(15 \%)$, and lumbosciatalgic in 8 patients $(11 \%)$. Fifty-six patients $(78 \%)$ had a single level of diskal compression, while multilevel compression was present in 16 patients (22\%). Levels involved by diskal protrusion were: L4-L5 intervertebral disk in 47 patients, L5-S1 in 15 patients, and L3-L4 in 10 patients. The duration of symptoms ranged from 6 to 18 weeks and averaged 12 weeks. Twenty-three patients had already undergone image-guided percutaneous infiltrations, the results of which were poor or completely inadequate.

\section{Statistical Analysis}

The outcomes were evaluated using a numeric scale from 0 to 10 assigned subjectively from patient's report on pain intensity (visual analog scale, VAS), with 0 being no pain and 10 being the most severe pain imaginable. Pain was scored before and after the procedure.

Follow-up was performed with VAS evaluation at 1, 3, 6, and 12 months after procedure and with spinal MRI at 3, 6, and 12 months after treatment.

The pretreatment and post-treatment (1, 3, 6, and 12 months after the procedure) data were compared using Student's $t$-test with a two-tailed comparison. Statistical significance was accepted if the $p$ value was equal to or less than 0.01 for continuous variables.

\section{Technique}

Before each procedure, the patient's imaging studies were evaluated in the radiology department and a general survey of relevant medical problems was performed. The procedure and associated potential complications were explained to the patient, and their prior informed consent was obtained.

Nucleoplasty was performed in the angiography room with the patient in a prone or semi-oblique position, under mild sedation. The involved disk space was localized under fluoroscopic guidance and the soft tissues, on the side of predominant pain, infiltrated with local anesthetic approximately $8-10 \mathrm{~cm}$ from the midline [7, $8]$.

Using a posterolateral extrapedicular approach, with a $45^{\circ}$ angle of inclination, a 17-gauge 6-inch-long Crawford type (ArthroCare, Sunnyvale, CA, USA) spinal introducer needle was inserted through the annulus fibrosus [4]. The proper needle position in the intersomatic spaces between L1 and L5, utilized to avoid the

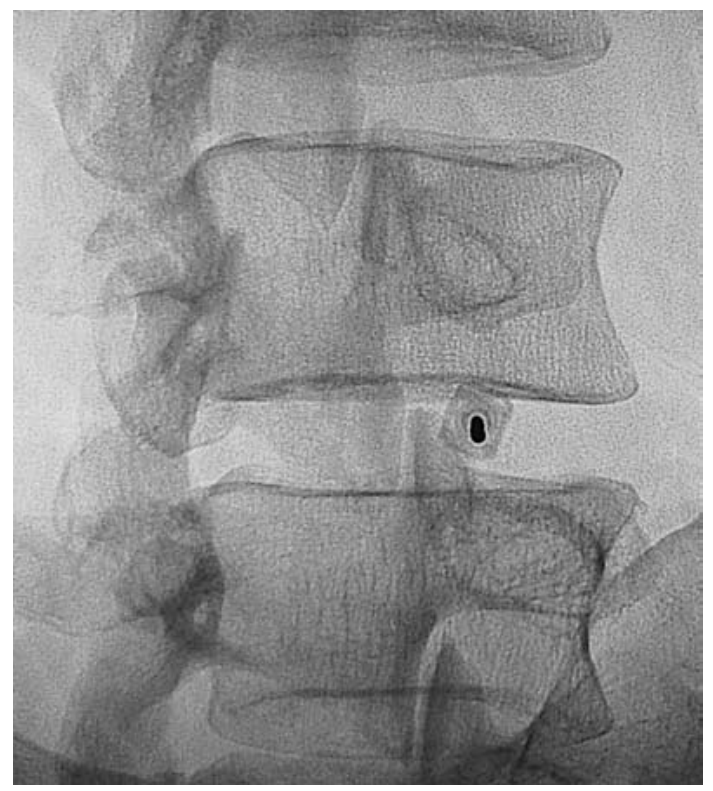

Fig. 1. Oblique fluoroscopic image: exact needle placement in the L4-L5 intersomatic space.

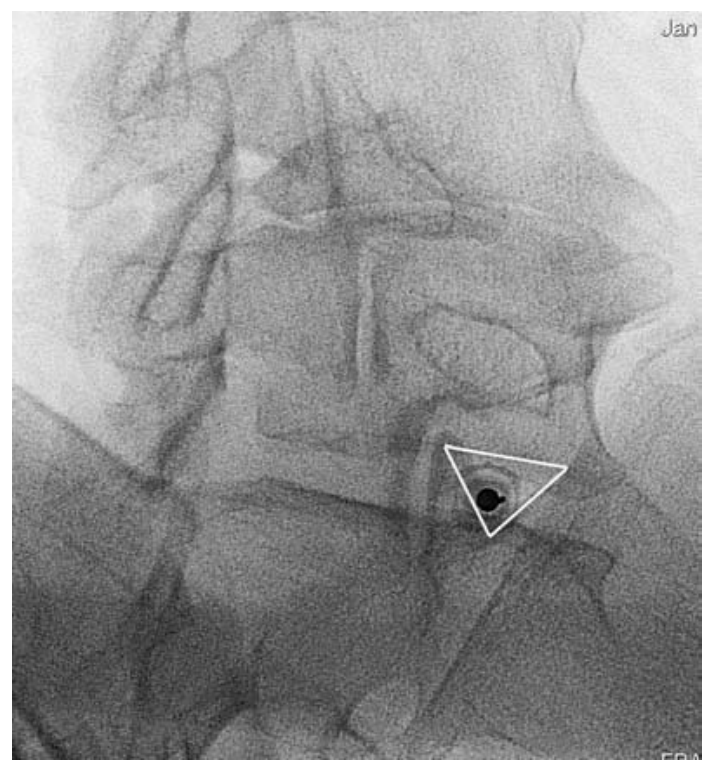

Fig. 2. Oblique fluoroscopic image: it is essential to establish the triangular target (white triangle) to obtain proper needle placement in the L5-S1 intersomatic space.

exiting nerve root, is, in the oblique projection, a few millimeters inferior and lateral to the superior pedicle and lateral to the superior articular process of the inferior vertebral level [9] (Fig. 1). In contrast the correct approach to the L5-S1 intersomatic space is, in the oblique projection, a triangular target site, bordered by the L5 inferior endplate superiorly, the S1 superior articular process posteromedially, and the iliac crest anterolaterally (Fig. 2). The needle tip must be positioned at the junction of the inner annulus and nucleus pulposus [8]. The anteroposterior projection was used 

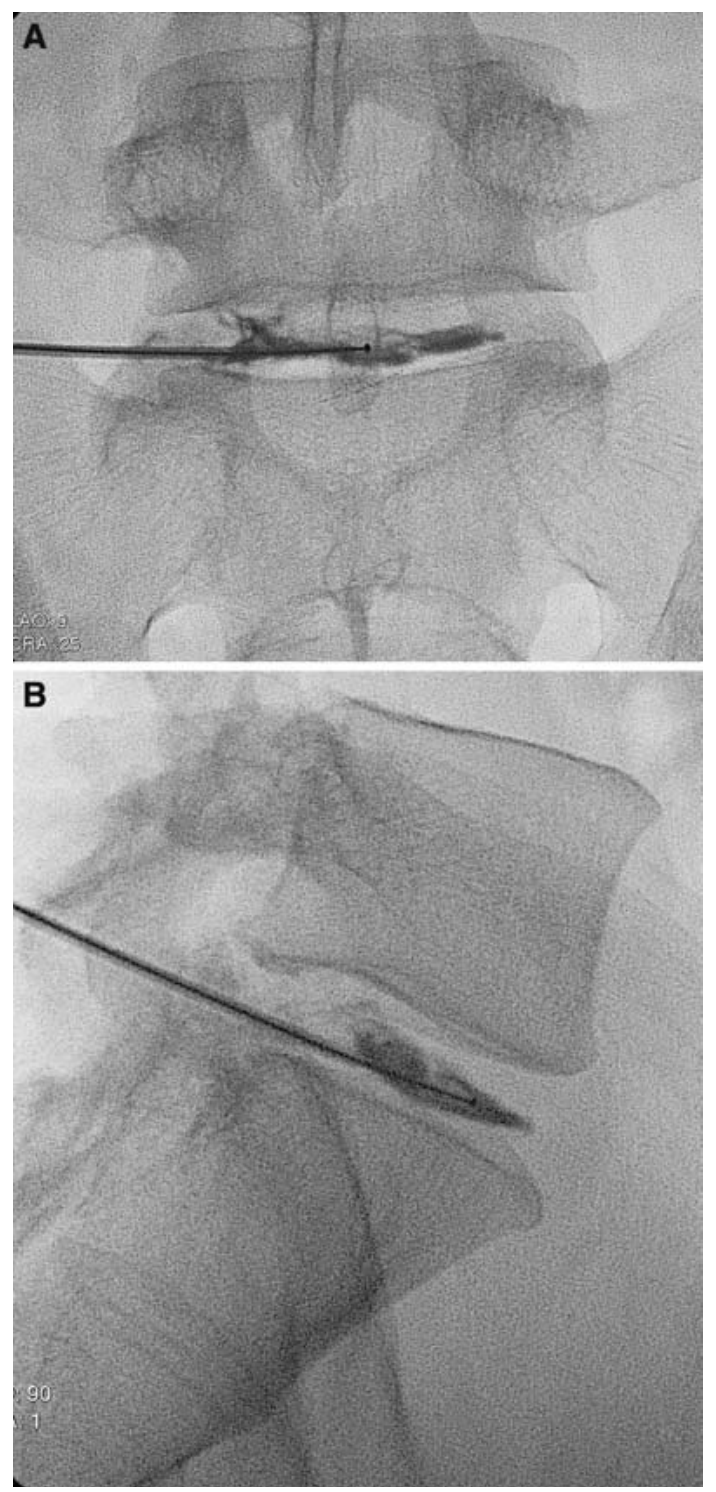

Fig. 3. A Anteroposterior and B lateral fluoroscopic images: positioning of the tip of the spinal wand in the nucleus pulposus.

to check the needle had not transgressed the transpedicular line, at a site just medial to the medial border of the pedicles above and below the disk space. The introducer remained in place within the outer annulus during the entire procedure, providing access for the spinal wand into the nucleus [4].

Through the needle we performed diskography, injecting contrast medium within the nucleus pulposus, in order to confirm the diagnosis of diskogenic pain with positive provocative elicitation of concordant pain as well as to verify the integrity of the annulus fibrosus [10-12].

A Perc-DLE spinal wand (ArthroCare, Sunnyvale, CA, USA) was advanced into the disk via the access cannula and placed with its tip approximately $5 \mathrm{~mm}$ beyond the edge of the cannula, ensuring that the active portion of the wand was beyond the inner layer of the annulus and was placed in the nucleus (Fig. 3). A circumferential reference mark on the shaft of the spinal needle
A
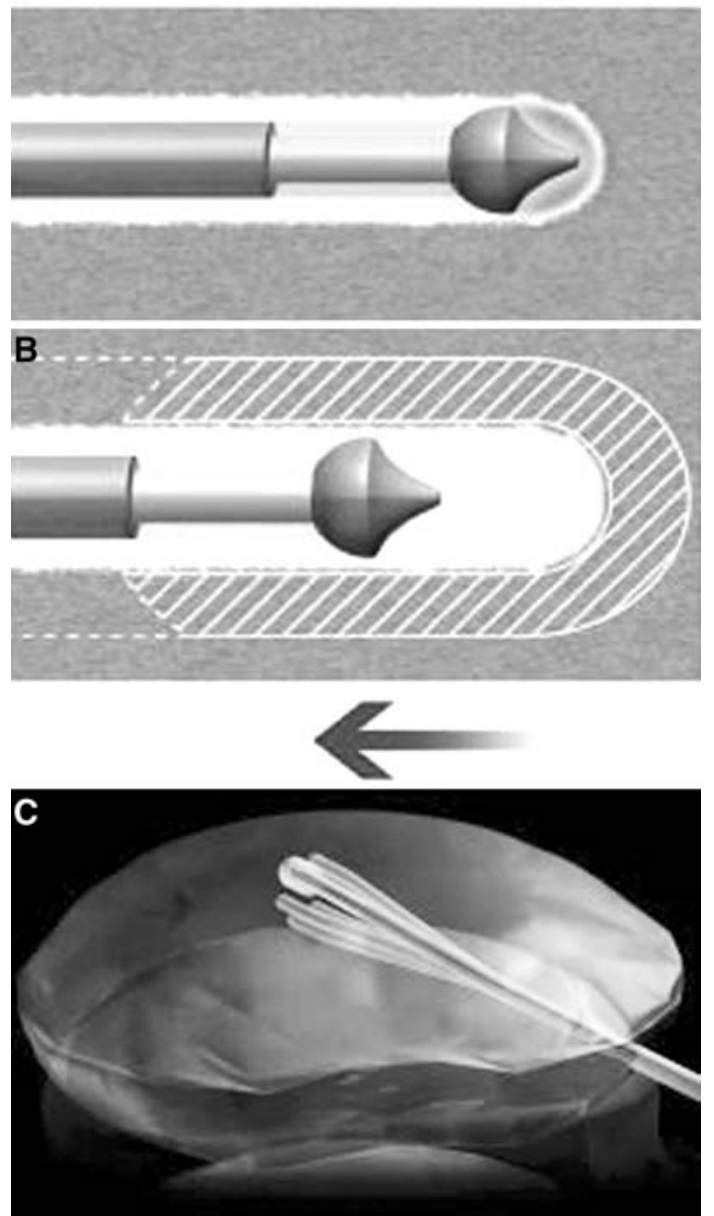

Fig. 4. A Ablation mode: advancing the wand tip has created a small defined channel in the nucleus pulposus. B Coagulation mode: withdrawing the wand tip has widened the channel previously created (approx. $1 \mathrm{~mm}$ radius). C Schematic drawing of the cone-shaped area of decompression generated in the nucleus pulposus. (Images provided by ArthroCare Inc., Sunnyvale, CA, USA.)

was placed adjacent to the entry site, marking the proximal channel limit. The wand was advanced until the inner surface of annulus on the opposite side, located under the lateral view, was between the anterior border of corresponding vertebral bodies. The depth stop marker on the shaft of the Perc-DLE wand was advanced close to the needle hub to designate the distal channeling limit. The wand is a $1 \mathrm{~mm}$ diameter, bipolar instrument designed for decompression of the disk nucleus utilizing coblation (tissue ablation "energy" and coagulation "heat"), and has a slight $C$ curve at the tip to allow for channeling [8]. The wand is connected to an Arthrocare System 2000 Controller radiofrequency generator (ArthroCare, Sunnyvale, CA, USA). This process generates a unique low-temperature plasma field, for precise, controlled ablation with minimal risk of thermal injury. Ablation, at a speed of $0.5 \mathrm{~cm} / \mathrm{sec}$, generates approximately 120 volts of energy at the tip of the wand with resultant temperatures of $50-70^{\circ} \mathrm{C}$ [13]. 

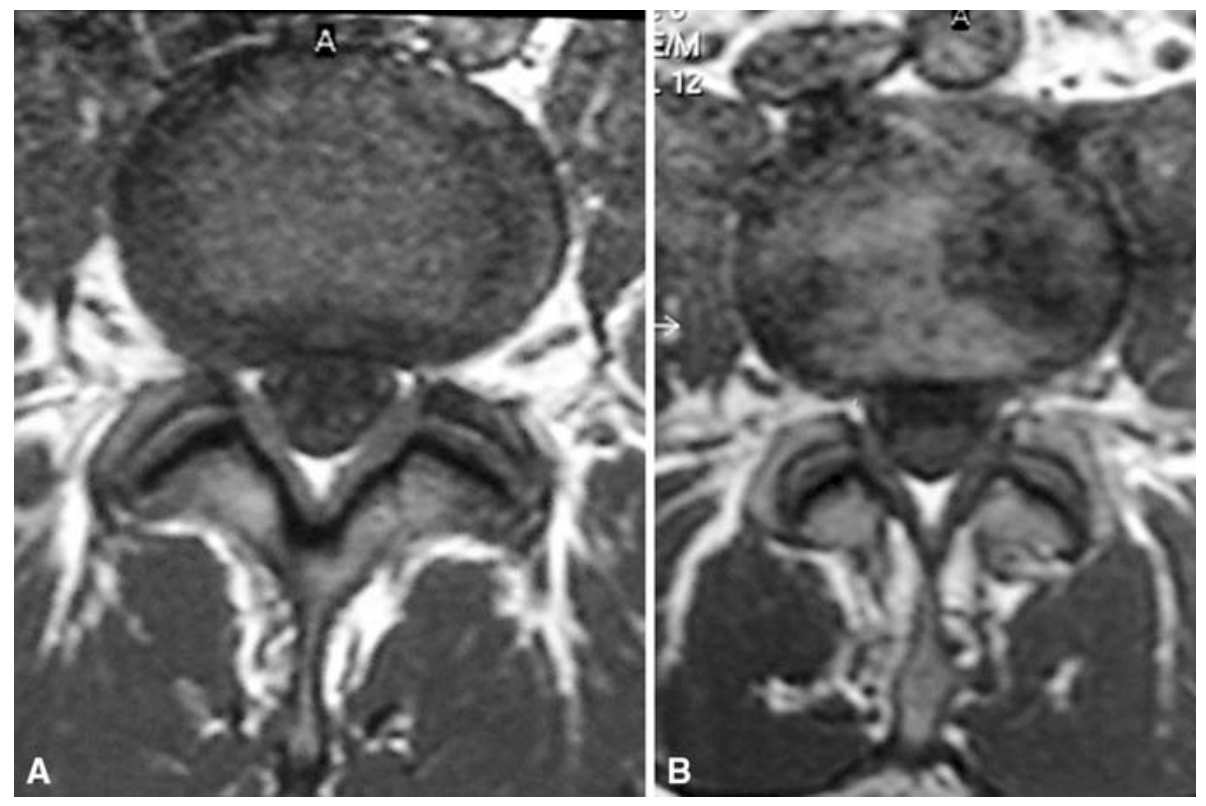

Fig. 5. $\mathrm{MR}$ images $\mathbf{A}$ before and $\mathbf{B}$ after treatment, showing clearly the reduction in the diskal lesion at 1 year follow-up.
The decompression process, advancing the wand in ablation mode, creates a channel from the posterolateral annulus to the anteromedial annulus [4] (Fig. 4A). On withdrawal of the wand to the proximal channel limit, the coagulation mode is used at 60 volts of energy and a tip temperature of $70^{\circ} \mathrm{C}$ [13]. On exit the thermal effect results in denaturation of the type II collagen and proteoglycans with resultant shrinkage of the surrounding collagen and widening of the channel (approximately $1 \mathrm{~mm}$ radius) (Fig. 4B).

The whole coblation procedure is performed under lateral projection. With clockwise rotation of the needle tip, a total of six channels are created at the 2, 4, 6, 8, 10, and 12 o'clock positions $[7,8]$. In every channel created by the needle tip, ablation with a mean duration of $8 \mathrm{sec}$ followed by $15 \mathrm{sec}$ of coagulation treatment is performed. Because of the C-shaped curve at the tip of the wand these six channels decompress a cone-shaped area of the nucleus (Fig. 4C).

The mean length of the procedure is $20 \mathrm{~min}$. The $1 \mathrm{~mm}$ skin incision is then closed with Steril-Strips and the patient placed supine on the bed for $2 \mathrm{hr}$. Postoperatively patients were allowed unlimited walking, standing, and sitting, but were instructed to limit any lifting, bending or stooping for 2 weeks. Return to sedentary or light work was permitted at 3-4 days following the surgery. Formal physical therapy with an emphasis on lumbar stabilization exercises started 3 weeks after the procedure [4].

\section{Results}

All patients were successfully treated without any significant complication during the procedure. No patient suffered pain during the coblation procedure. The hospital stay ranged from 2 to 4 days, with an average of 3 days. The mean follow-up of the study was 18 months (range 12-21 months); 2 patients were lost to 1 year follow-up.

The average preprocedural pain level for all patients was reported as 8.2 while the average pain level 1 at the 12 month follow-up was 4.1. The VAS scores in 70 patients after 12 months of follow-up demonstrated that 17\% (12 patients) were completely satisfied with the treatment (mean score 0-2) and had complete resolution of symptoms, $62 \%$ (43 patients) obtained a good result (mean score 3-4), 19\% (13 patients) received little benefit (mean score 5-7), and in $3 \%$ ( 2 patients) results were completely unsatisfactory and necessitated surgical treatment (mean score 8-9).

At 1 year, $79 \%$ of patients demonstrated a statistically significant improvement in numeric pain scores, defined as a decrease of at least 4 VAS units (95\% confidence interval $70.5-86.5 \%, p<0.01)$. The 1 year follow-up MRI examination confirmed the clinical results, demonstrating a reduction in the lesions in almost $80 \%$ of patients (Fig. 5).

\section{Discussion}

Chronic low back pain is the most common ailment in modern industrial societies. It ranks first among musculoskeletal disorders, resulting in serious financial and social consequences. The intervertebral disk, because of its highly specialized role and relatively susceptible nature, is one of the major sources of low back pain syndrome [1-14]. This injury can be accompanied by intense pain around the affected disk as well as pain that radiates to the lower back and legs $[15,16]$.

It is uncertain whether diskogenic pain is mediated via chemical, mechanical or neural mechanisms, or a combination of these $[2,17,18]$. Hydrostatic pressure, between the disk and vertebral endplates, plays a very important role in the regulation of nutrient supply to the disk and in removal of waste from the cells of the nucleus pulposus, which is an avascular structure. With aging, disease or injury, the structures through which nutrients diffuse may become less permeable to the essential blood supply, forcing more of the disk to function anaerobically. As disk degeneration pro- 
gresses, volume loss occurs within the nucleus pulposus due to a decrease in proteoglycan and water concentration. Due to the lack of nutrients and oxygen, the cells are forced to metabolize anaerobically, generating large amounts of lactic acid, increasing acidity and further degrading the intradiskal matrix. The strength of the lumbar disk depends on fluid exchange and a balance between proteoglycan synthesis and breakdown within the matrix. As these components decrease, the applied load is transferred to the annulus and posterior elements of the spine. This transfer greatly increases the probability of annular tear and/or herniation [19]. The outer rim of the annulus is innervated by the meningeal branch of the recurrent sinuvertebral nerve, as well as the rami communicantes from multiple superior and inferior dorsal root ganglia. Outside the disk, the anterior and posterior longitudinal ligaments, which may be stretched by herniation or chemically irritated by the release of inflammatory chemicals from within the disk, are also richly innervated, providing another potential source of pain [2022].

Treatment of diskogenic low back pain is based on the theory that a small reduction in disk volume, involving removal of part of the nucleus via surgical or minimally invasive methods, can result in a large change in intradiskal pressure [23]. Surgical treatments for intervertebral disk herniation consist of open laminectomy, diskectomy, and microdiskectomy. Minimally invasive methods involve automated percutaneous lumbar diskectomy, arthroscopic microdiskectomy, chemonucleolysis, YAG laser diskectomy, intradiskal electrothermal annuloplasty (IDET), oxygen-ozone therapy, and nucleoplasty [24, 25]. Surgical treatments such as open laminectomy, diskectomy, and microdiskectomy are often targeted at patients with uncontained or large herniations, and/or sequestered disks. Patients presenting with small contained herniated disks who have not responded to conservative noninvasive treatment, are often not considered as surgical candidates [26].

Annular integrity may be another important variable in achieving a more beneficial outcome for patients undergoing disk decompression. Traditional microdisketomy procedures that create an incision or "annulotomy" in the outer fibrous rings of the herniated disk may, given that annular repair occurs very gradually, weaken or reduce intervertebral disk stability and cause disk reherniation and subsequent reoperations. Another important factor affecting the outcome after surgery is the formation of adhesions and scar tissue. Adhesions between the posterior annulus and the nerve root (failed back syndrome) are common following diskectomies [27-30].

The general trend in spinal surgery, as in all surgical treatment, toward reduction of devices and minimization of surgery-related trauma, led to the development of numerous minimally invasive percutaneous techniques for disk decompression and back pain treatment. Chemonucleolysis, consisting of intradiskal chymopapain injection, available since 1964 [31], demonstrated long-term success rates between $66 \%$ and $88 \%$. Unfortunately, chymopapain had the potential risk of paralysis secondary to transverse myelitis and an anaphylaxis rate estimated at $0.3-0.5 \%$. These risks far outweighed the potential benefit of this procedure [31].

In 1984 an alternative method for percutaneous disk removal was proposed, using automated percutaneous lumbar diskectomy, a technique that utilizes a $20.3 \mathrm{~cm}$ needle inserted through a $3 \mathrm{~mm}$ cannula. In a large series of percutaneous diskectomy patients there was a $75 \%$ success rate of good to excellent outcomes. Automated percutaneous lumbar diskectomy further supported the previous conclusions that the outcome of disk excision was independent of the quantity of disk material removed [32, 33].

Also in 1984, Choy et al. [34] reported on YAG laser diskectomy, a system based on intradiskal pressure reduction using laser energy introduced through a needle in the nucleus pulposus. This yielded success rates ranging between $63 \%$ and $89 \%$, with pain relief lasting over 12 years. However, disadvantages of this technique included moderate to severe intraoperative pain secondary to the thermal effect of the laser, postoperative low back pain and spasm, and inability to visualize the tip of the laser beam under fluoroscopy [34, 35].

Intradiskal electrothermal annuloplasty (IDET), first performed in 1998, follows the concept of "annuloplasty," in which thermal heating of the annulus seals annular tears and denervates the outer one third of the annulus by coagulation of the type $\mathrm{C}$ afferent nerve fibers [6, 8, 36]. A curved thermal resistance catheter is coiled within the disk under fluoroscopic guidance. The wire is then heated to $90^{\circ} \mathrm{C}$, and maintained at that temperature for at least $4 \mathrm{~min}$. In theory this should produce denaturing of collagen fibers arranged in a triple helix, forming random coils with intermittent cross-links and resulting in contraction of collagen fibers. However, studies with thermocouples verified that during the IDET procedure annular temperatures never reach $70^{\circ} \mathrm{C}$ and only sporadically reach $45^{\circ} \mathrm{C}$, which is insufficient to destroy afferent nerve fibers and shrinkage collagen fibers. Other disadvantages are the potential risk of annular perforation, due to technical difficulties involved in threading a curved $30 \mathrm{~cm}$ wire around the annulus, and the amount of intraoperative pain experienced by patients during annulus heating [37].

Oxygen-ozone therapy exploits the chemical properties of ozone, an unstable allotropic form of oxygen. The therapeutic aims of intradiskal and periganglionic injection of an oxygen-ozone mixture is degeneration of proteoglycans of the nucleus pulposus, with disk shrinkage and nerve root decompression, associated with analgesic and anti-inflammatory effects, which may counteract disk-induced pain [38, 39].

Percutaneous disk decompression (PDD) using coblation technology (nucleoplasty) is a new therapeutic option, used for orthopedic arthroscopic procedures since the mid-1990s and approved for use in the spine in July 2000. Nucleoplasty 
is based on the principle that a small reduction in volume in a closed hydraulic space, such as an intact disk, results in a disproportionately large fall in pressure [23]. Coblation of the nucleus pulposus content causes disk shrinkage with a vacuum effect, able to relieve pressure from the roots-a result called "implosion theory." This decompression leads to higher axonal, liquoral, and hematic flow rates, bringing about a resolution of the periradicular inflammatory mechanism and better endorphin diffusion. The nucleoplasty technique implements this principle of diskal volumetric reduction through a controlled low-temperature ablative process $\left(40-70^{\circ} \mathrm{C}\right)$ in which heat generated is consumed in the plasma layer by the ionization process and tissue heating is minimal. The result is a gentle removal effect on target tissue with minimum dissolution effects on surrounding vital structures $[5,40]$. Preserving the integrity of these tissues should maintain the flow of nutrients to the cells of the nucleus pulposus, resulting in an increased degree of cellular rejuvenation following the procedure [41, 42]. Coblation technology involves the use of radiofrequency energy, to excite the electrolytes in a conductive medium, such as saline solution, creating a precisely focused plasma $1 \mathrm{~nm}$ thick at the tip of the wand. The plasma's energized particles have sufficient energy to break molecular bonds within the nucleus pulposus, dissolving the nuclear material through molecular dissociation, with the production of elemental molecules and low-molecular-weight gases. These byproducts (oxygen, nitrogen, hydrogen, carbon dioxide, etc.) exit the disk through the introducer needle, with approximately $1 \mathrm{~cm}^{3}$ of tissue volume being removed. In addition the water binding capacity of the matrix proteoglycan is reduced.

In our preliminary experience we obtained an overall success rate of $79 \%$. This successful response to therapy could be the result of elastic recoil of the disk that leads to a new conformation of the disk-nerve compartment. As in most disk percutaneous ablation therapies, it is important that the procedure should not be used in a poorly hydrated disk, considering the inadequate results in these patients.

The 2 patients who underwent surgery were treated with a conventional surgical procedure without any complication or variation as a result of the previous nucleoplasty.

In our experience nucleoplasty might be considered a valid alternative to conventional surgery, though good results are probably dependent on strict inclusion criteria and proper patient selection. The results of the present study must certainly be confirmed by larger series and compared with other different techniques with long-term follow-up. At present there are no large series studies that have evaluated nucleoplasty, so while the comparison with other studies is awaited, this minimally invasive procedure must be considered a very effective and safe technique for the treatment of patients with symptomatic contained disk herniation who present diskogenic axial back pain and/or leg pain, have failed conservative therapies, and are not considered candidates for open surgery.

\section{References}

1. Olmarker K, Rydebik B (1991) Pathophysiology of sciatica. Orthop Clin North Am 22:223-234

2. Kirkald-Willis WH (1999) Pathology and pathogenesis of the low back pain. In: Bernard T Jr (ed) Managing low back pain, 4th edn. Churchill Livingstone, Philadelphia, pp 65-95

3. Dworkin GE (2002) Advanced concepts in interventional spine care. J Am Osteopath Assoc 102S:8-11

4. Nardi PV, Cabezas D, Cesaroni A (2005) Percutaneous cervical nucleoplasty using coblation technology: Clinical results in fifty consecutive cases. Acta Neurochir Suppl 92:73-78

5. Alexandre A, Coro L, Azuelos A, Pellone M (2005) Percutaneous nucleoplasty for discoradicular conflict. Acta Neurochir 92:83-86

6. Cohen SP, Williams S, Kurihara C, Griffith S, Larkin TM (2005) Nucleoplasty with or without intradiscal electrothermal therapy (IDET) as a treatment for lumbar herniated disc. J Spinal Disord Tech 18S: $119-124$

7. Marin FZ (2005) CAM versus nucleoplasty. Acta Neurochir Suppl 92S:111-114

8. Welch WC, Gerszten PC (2002) Alternative strategies for lumbar discectomy: Intradiscal electrothermy and nucleoplasty. Neurosurg Focus 13:E7

9. Kelekis AD, Somon T, Yilmaz H, et al. (2005) Interventional spine procedures. Eur J Radiol 55:362-383

10. Bogduk N (1995) Discography. In: Aprilo C, Darby R, White AH, Schofferman JA (eds) Spine care. Mosby-Year Book, St Louis, pp 219239

11. Fortin J (1995) Lumbar and thoracic discography with CT and MRI correlations. In: Lennard TA (ed) Physiatric procedures and clinical practice. Hanley \& Belfus, Philadelphia

12. Carragee EJ, Lincoln T, Parmar VS, Alamin T (2006) A gold standard evaluation of the "discogenic pain" diagnosis as determined by provocative discography. Spine 31:2115-2123

13. Nau WH, Diederich CJ (2004) Evaluation of temperature distributions in cadaveric lumbar spine during nucleoplasty. Phys Med Biol 49:1583-1594

14. Weinstein J (1990) Recent advances in the neurophysiology of pain. Phys Med Rehabil: State of the Art Reviews 4:201-220

15. Manchikanti L, Pampati V, Fellows B, et al. (2001) Characteristics of chronic low back pain in patients in an interventional pain management setting: A prospective evaluation. Pain Physician 4:131-142

16. Nachemson AL (1976) Advances in low-back pain. Clin Orthop Relat Res Nov 200:266-278

17. Saal JA (1990) The role of inflammation in lumbar pain. Phys Med Rehabil: State of the Art Reviews 4:191-200

18. Woodward J (1995) Epidural procedures in spine pain management. In: Lennard TA (ed) Physiatric procedures and clinical practice. Hanley \& Belfus, Philadelphia, pp 260-291

19. Dreyfuss P (1995) Zygapophyseal joint injection techniques in the spinal axis. In: Lennard TA (ed) Physiatric procedures and clinical practice. Hanley \& Belfus, Philadelphia

20. Manchikanti L, Pampati V, Bakhit CE, et al. (2001) Effectiveness of lumbar facet joint nerve blocks in chronic low back pain: A randomized clinical trial. Pain Physician 4:101-117

21. Manchikanti L, Pampati V, Fellows B, Rivera J, Beyer CD, Damron KS (2001) Role of one day epidural adhesiolysis in management of chronic low back pain: A randomized clinical trial. Pain Physician 4:153-166

22. Slipman CW, Zacharia I (2001) The role of diagnostic selective nerve root blocks in the management of spinal pain. Pain Physician 4:214-226

23. Choy DSJ, Ascher PW, Saddekni S, et al. (1992) Percutaneous laser disc decompression: A new therapeutic modality. Spine 17:949-956

24. Andreula C, Muto M, Leonardi M (2004) Interventional spinal procedures [review]. Eur J Radiol 50:112-119

25. Saal JA (1990) Intervertebral disc herniation: Advances in nonoperative treatment. Phys Med Rehabil: State of the Art Reviews 4:214227

26. Schrieber A, Suezawa Y (1986) Transdiscoscopic percutaneous nucleotomy in disc herniation. Orthop Rev 15:75-78

27. Onesti ST (2004) Failed back syndrome [review]. Neurologist 10:259264 
28. Anderson PA, Rouleau JP (2004) Intervertebral disc arthroplasty. Spine 29:2779-2786

29. Schwarzer AC, Aprill CN, Derby R, Fortin J, Kine G, Bogduk N (1995) The prevalence and clinical features of internal disc disruption in patients with chronic low back pain. Spine 20:1878-1883

30. Sprangfort EV (1972) The lumbar disc herniation: A computer aided analysis of 2504 operations. Acta Orthop Scand 142:1-93

31. Smith L (1964) Enzyme dissolution of the nucleus pulposus in humans JAMA 187:137-140

32. Gill K (1993) Percutaneous lumbar discectomy. Am Acad Orthop Surg $1: 33-40$

33. Kamlin P, Brager MD (1987) Percutaneous posterolateral discectomy: Anatomy and mechanism. Clin Orthop 223:145-154

34. Choy DSJ, Case RB, Fielding JW, et al. (1987) Percutaneous laser nucleolysis of the lumbar disc. N Engl J Med 317:771-772

35. Liebler WA (1995) Percutaneous laser disc nucleotomy. Clin Orthop 310:58-66

36. Saal JA, Saal JS (2000) Intradiscal electrothermal treatment for chronic discogenic low back pain: A prospective outcome study with minimum 1-year follow-up. Spine 25:2622-2627
37. Freeman BJ, Fraser RD, Cain CM, Hall DJ, Chapple DC (2005) A randomized, double-blind, controlled trial: Intradiscal electrothermal therapy versus placebo for the treatment of chronic discogenic low back pain. Spine 30:2369-2377

38. Andreula CF, Simonetti L, De Santis F, Agati R, Ricci R, Leonardi M (2003) Minimally invasive oxygen-ozone therapy for lumbar disk herniation. AJNR Am J Neuroradiol 24:996-1000

39. Muto M, Andreula C, Leonardi M (2004) Treatment of herniated lumbar disk by intradiscal and intraforaminal oxygen-ozone $\left(\mathrm{O}_{2}-\mathrm{O}_{3}\right)$ injection. J Neuroradiol 31:183-189

40. Wang JC, Kabo JM, Tsou PM, Halevi L, Shamie AN (2005) The effect of uniform heating on the biomechanical properties of the intervertebral disc in a porcine model. Spine J 5:64-70

41. Chen YC, Lee SH, Saenz Y, Lehman NL (2003) Histologic findings of disc, end plate and neural elements after coblation of nucleus pulposus: An experimental nucleoplasty study. Spine J 3:466-470

42. Chen YC, Lee SH, Chen D (2003) Intradiscal pressure study of percutaneous disc decompression with nucleoplasty in human cadavers. Spine 28:661-665 